\title{
The influence of water content and ionicity on the efficacy of soft contact lens care regimens on Pseudomonas aeruginosa
}

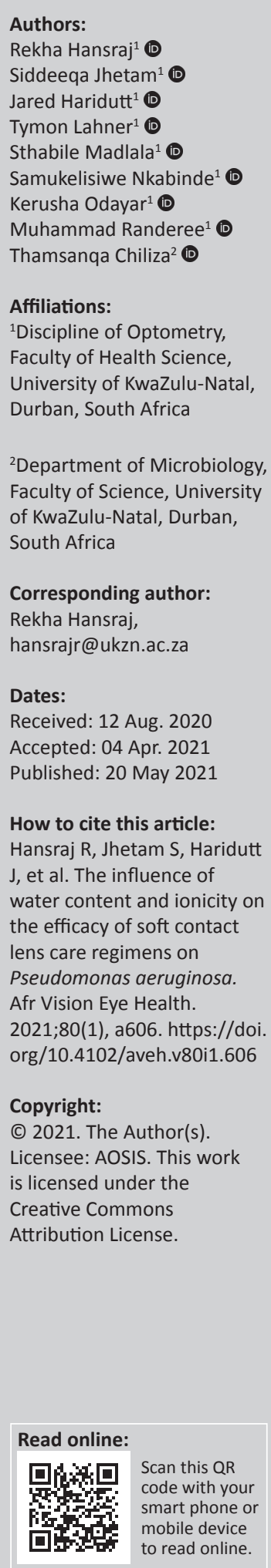

Background: Contact lens care regimens appear to be prescribed based on familiarity or by matching contact lens brands rather than consideration to efficacy.

Aim: This study compared the effectiveness of multipurpose and peroxide cleaning solutions on low- and high-water content ionic and non-ionic soft contact lenses, in removing Pseudomonas aeruginosa (P. aeruginosa).

Setting: The laboratory work was conducted by health science students in a graduate level programme in the microbiology laboratory at a university in south-eastern South Africa.

Methods: A quantitative analytical experimental design was used. Four groups (labelled I, II, III and IV) of soft contact lenses, varied ionicity and water content were inoculated with $P$. aeruginosa and then exposed to three solutions containing antimicrobial ingredients, polyaminopropyl biguanide (Dymed), hydrogen peroxide $\left(\mathrm{H}_{2} \mathrm{O}_{2}\right)$ and polyquaternium-1 (Polyquad). Each group contained 14 soft contact lenses, with the exception of Group III in which eight lenses were used. Saline served as the control. After $18 \mathrm{~h}$, the remaining colonyforming units were counted using visual inspection as an indicator of efficacy against P. aeruginosa.

Results: The solution containing $\mathrm{H}_{2} \mathrm{O}_{2}$ was found to be the most effective in removing P. aeruginosa from all Food and Drug Administration (FDA) groups of contact lenses whilst Dymed was found to be comparatively ineffective for this organism. Water content and ionicity had no effect on the efficacy of the solutions.

Conclusion: Practitioners should consider the efficacy of the active ingredients against microorganisms when dispensing contact lens solutions, particularly for contact lens wearers at risk for $P$. aeruginosa infections.

Keywords: water content; ionicity; contact lens solutions; soft contact lens; Dymed; hydrogen peroxide; Polyquad; P. aeruginosa.

\section{Introduction}

Soft contact lenses, including hydrogel but more so silicone hydrogel, are increasingly being used for the correction of refractive errors. ${ }^{1}$ The effective and safe use of soft contact lenses are dependent on an optimal fit, correct insertion and removal technique, as well as good care and hygiene of the lenses. ${ }^{2}$ The care regimen of contact lenses should include a thorough disinfection routine with an appropriate contact lens cleaning solution. The purpose of a contact lens solution is to disinfect, clean and hydrate the contact lens primarily to reduce the microbial load that enters into the eye and ensure safe wear and optimal vision. ${ }^{3}$ Generally, contact lens solutions can either be categorised as multipurpose or peroxide disinfecting systems. ${ }^{3}$ The mode of action of most multipurpose systems is to disrupt the microbial membranes by negating their selective barrier effect which leads to the death of microbes. ${ }^{3,4}$ Hydrogen peroxide $\left(\mathrm{H}_{2} \mathrm{O}_{2}\right)$, on the other hand, is a strong oxidising agent which disrupts the metabolism of proteins, lipids and DNA in the microorganism, thereby resulting in cell death. ${ }^{3,4}$ Inefficient hygiene and care of contact lenses related to either the cleaning technique or efficacy of the cleaning solution is strongly related to microbial contamination of soft contact lenses which can consequently lead to eye infections often involving the cornea..$^{5}$ Infections can include bacterial, fungal or amoebic keratitis that lead to inflammation of the cornea and if not treated promptly may cause impaired vision or even blindness. ${ }^{6}$ 
Bacterial keratitis is one of the most serious complications of a compromised corneal epithelium particularly in contact lens wearers with Staphylococci, Streptococci and Pseudomonas aeruginosa (P. aeruginosa) often being the associated organisms. Pseudomonas aeruginosa, an opportunistic gram negative bacteria, is reported as the most common cause of bacterial keratitis in contact lens wearers ${ }^{7,8,9,10}$ as it is able to easily adhere to the surface of contact lenses by means of a biofilm which forms within $24 \mathrm{~h} \cdot 5,8,11,12$ The incidence of microbial keratitis linked to $P$. aeruginosa and contact lens wear has been reported as 13.04 cases per 10000 individuals a year with the pathogen being identified as the causative agent in $70 \%$ of cultured contact lens associated microbial keratitis. ${ }^{6,8}$ Pseudomonas aeruginosa may also display an innate or acquired resistance to cleaning solutions ${ }^{13,14}$, particularly because of its strong adhesion to lenses linked to its surface hydrophobicity. ${ }^{15}$ The higher incidence of keratitis with this pathogen, therefore, has been attributed to its virulent features as well as its resistance in removal from contact lenses. ${ }^{13,16}$ This emphasises the need for contact lens disinfection solutions to have superior efficacy against $P$. aeruginosa.

Contact lens solutions are formulated to combat and protect against many pathogens. The effectiveness of the solutions has been found to depend on certain lens properties including water content and ionicity ${ }^{17,18}$ which influence bacterial adhesion to lenses. ${ }^{15}$ Water content refers to the proportion of water in the contact lens or the percentage of water uptake which is dependent on the chemical structure and formulation of the lens. ${ }^{17,18}$ Oxygen permeability is expected to increase with an increase in water content with hydrogels but in the case of silicone hydrogels, this has not always been the case. ${ }^{19,20}$ This is important as there is a greater risk of infection and lens contamination when the cornea does not receive the required amount of oxygen resulting in hypoxia. However, when compared to low water content lenses, high water content lens tend to be more prone to deposits and thus microorganism adherence. ${ }^{18,21}$ In contrast, Miller and Ahearn ${ }^{22}$ reported $P$. aeruginosa to have decreased adherence to high water content lenses.

Ionicity of a soft contact lens refers to the surface charge of the lens, ${ }^{23}$ and soft lenses may be classified as either ionic or non-ionic. The ionicity of a soft contact lens can affect how quickly protein deposits are formed on the lenses during wear. ${ }^{23}$ Ionic materials have a negatively charged surface and therefore may attract positively charged tear proteins, resulting in increased deposit formation. ${ }^{18}$ Non-ionic materials are treated to reduce this negative surface charge and may, therefore, be less prone to attract protein deposits. ${ }^{23}$ Gopinathan et al., ${ }^{6}$ however, found that ionicity of a contact lens has little to no effect on the risk of contamination.

Many of the previous studies that assessed the disinfectant capability of contact lens solutions against $P$. aeruginosa did not involve contact lenses ${ }^{9,24,25,26,27}$ whilst others that involved contact lenses did not include peroxide solutions. ${ }^{5}$ No study was found that investigated the effect of water content and ionicity on the efficacy of a contact lens solution in removing $P$. aeruginosa despite findings of variation in adherence of microbes based on these properties. The findings of this study may assist practitioners in prescribing the most effective contact lens solutions for the patient and thereby reduce the risk of infection specifically related to this specific microbe.

\section{Methods \\ Study design}

The study used an analytical quantitative experimental design and was a collaborative study between optometry and microbiology. Data collection commenced once ethical clearance was obtained from the relevant authorities.

\section{Study population and sampling strategy}

Non-random purposive sampling was used to select the cleaning solutions and contact lenses. Three, no-rub, cleaning systems were chosen and included either hydrogen peroxide $\left(\mathrm{H}_{2} \mathrm{O}_{2}\right)$, PolyQuad (polyquaternium-1) or Dymed (polyaminopropyl biguanide) as the antimicrobial agent. Saline served as a control. The total number of lenses from each Food and Drug Administration (FDA) group is shown in Table 1.

\section{Study setting and data collection}

Pseudomonas aeruginosa from the culture collection at the microbiology laboratory was grown on nutrient agar at $37^{\circ} \mathrm{C}$ overnight. Single colony was grown in $200 \mathrm{~mL}$ of nutrient broth to the log phase growth (optical density [OD] of 0.5 at $595 \mathrm{~nm}$ ) and the $P$. aeruginosa concentration was adjusted using a spectrophotometer (Bio-Rad, Hercules, CA) at $595 \mathrm{~nm}$ to obtain an OD of $0.1\left(1 \times 10^{8}\right.$ colony-forming unit $(\mathrm{CFU}) / \mathrm{mL})$. Different types of contact lenses were incubated in separate petri dishes with $15 \mathrm{~mL}$ of $\log$ phase P. aeruginosa culture $\left(1 \times 10^{8} \mathrm{CFU} / \mathrm{mL}\right)$ in saline solution for $180 \mathrm{~min}$ at $37^{\circ} \mathrm{C}$ by shaking.

The lenses were removed and each lens was placed in a well with appropriate contact lens cleaning solution as well as in saline and incubated for $\sim 18 \mathrm{~h}$. Then each lens was removed and placed in a test tube with $2 \mathrm{~mL}$ of sterile saline. The test tube was vortexed for $45 \mathrm{~s}$ and $200 \mu \mathrm{L}$ was plated onto a nutrient agar plate. The negative control not exposed to $P$. aeruginosa culture was also plated. The agar plates were then incubated at $37^{\circ} \mathrm{C}$ overnight. The following day, the remaining visible colony units were then counted using

TABLE 1: Contact lenses selected for the study, with number of lenses used per Food and Drug Administration group.

\begin{tabular}{llll}
\hline FDA group & Water content (\%) & lonicity & Material \\
\hline I $(n=14)$ & 24 (low) & Non-ionic & Lotrafilcon A \\
II $(n=14)$ & 62 (high) & Non-ionic & Omafilcon A \\
III $(n=8)$ & 36 (low) & Ionic & Balafilcon A \\
IV $(n=14)$ & 55 (high) & Ionic & Methafilcon A \\
\hline
\end{tabular}

FDA, Food and Drug Administration. 
visual inspection and averaged. Analysis of variance (ANOVA) was used to investigate differences between groups at a $95 \%$ level of significance.

\section{Ethical considerations}

Full ethical clearance was obtained by the Humanities and Social Science Research and Ethics Committee of the University of KwaZulu-Natal (HSS/1120/016U).

\section{Results}

In Figures 1, 2 and 3, the $y$-axis upper limit has been set to 50 to allow a better representation of the smaller values of the average number (no.) of CFUs remaining for the solutions, however, the approximate number is shown above each respective column. The solutions are labelled in terms of their active ingredients. Figure 1 shows the average number of CFUs remaining in each FDA group of contact lenses following exposure to each of the three different contact lens solutions and the control (saline).

The CFUs remaining in all FDA groups following exposure to both saline and the solution containing Dymed fell in the category 'Too many to count', that is, greater than 1000 CFUs remaining on the lens. Following exposure to the solution containing peroxide $\left(\mathrm{H}_{2} \mathrm{O}_{2}\right)$, no CFUs remained in any FDA group of lenses. A few CFUs remained in FDA Groups I, II and IV, following exposure to the solution containing Polyquad whilst no CFUs remained on Group III lenses.

Figure 2 illustrates a comparison of the average no. of CFUs remaining on non-ionic and ionic soft contact lenses after exposure to each of the solutions. No significant difference (one-way ANOVA, $p>0.05$ ) was found in the average number of CFUs remaining on either non-ionic or ionic lenses after exposure to each of the solutions.

Figure 3 shows the average no. of CFUs remaining on high water content lenses versus low water content lenses following exposure to each of the solutions. There appeared to be no significant difference (ANOVA, $p>0.05$ ) in the remaining CFUs when comparing high water content to low water content lenses irrespective of the solution used.

\section{Discussion}

Varied efficacy in disinfecting the contact lenses from $P$. aeruginosa was observed with the different solutions analysed. The solution containing $\mathrm{H}_{2} \mathrm{O}_{2}$ was the most effective as no CFUs of the microorganism concerned remained on the soft contact lenses, irrespective of the FDA grouping, following disinfection. The Polyquad-based solution was the second most effective for all four soft contact lens types whilst the Dymed-based solution appeared to be the least effective against $P$. aeruginosa. These findings may be related to the difference in mechanisms of action of peroxide systems when compared to chemical systems with oxygen releasing solutions and peroxide systems having been reported to be

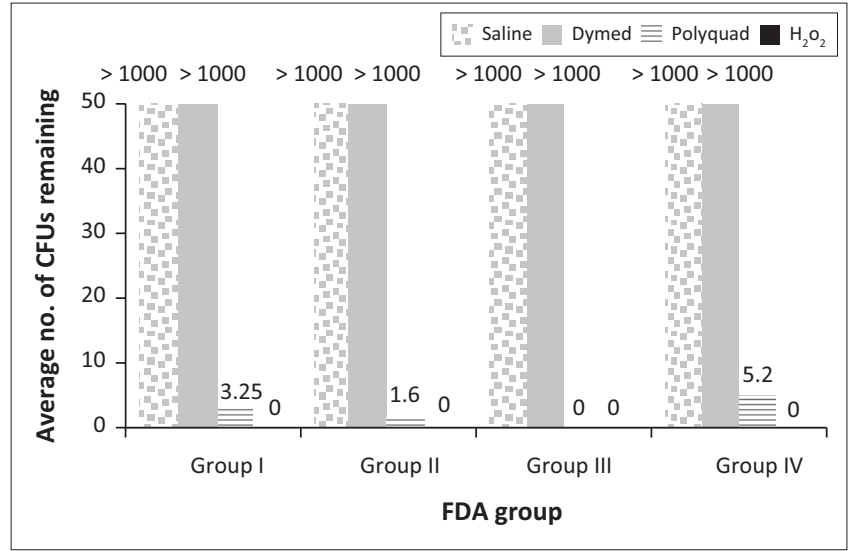

FDA, Food and Drug Administration; CFU, colony forming units.

FIGURE 1: Solution effectiveness on the different Food and Drug Administration groups in removing Pseudomonas aeruginosa.

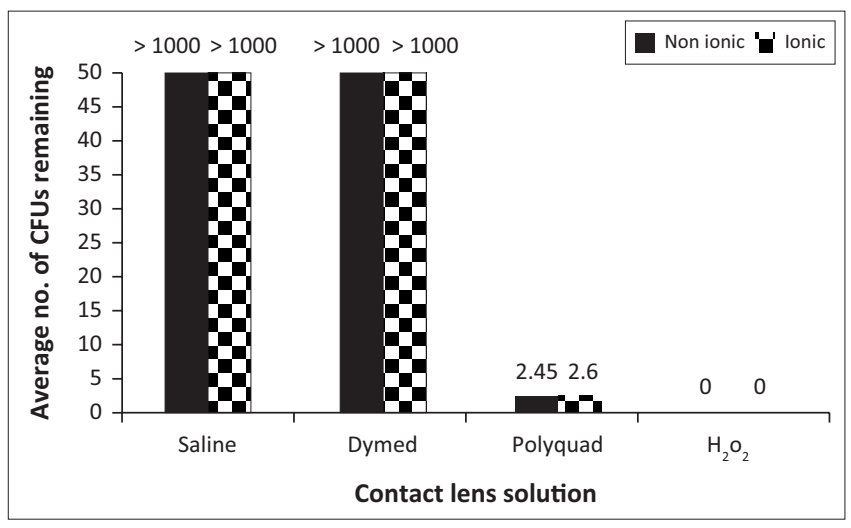

$\mathrm{CFU}$, colony-forming units.

FIGURE 2: Solution effectiveness on non-ionic versus ionic soft contact lenses in removing Pseudomonas aeruginosa.

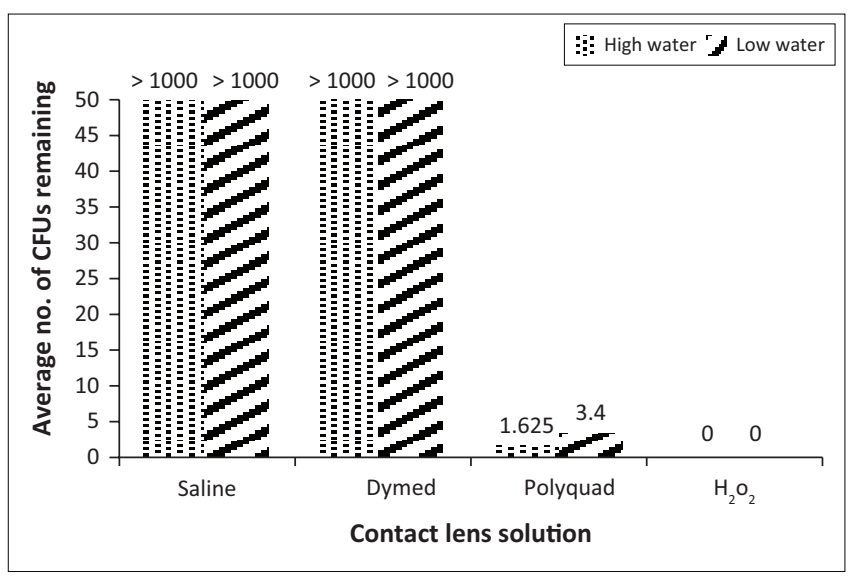

CFU, colony-forming units.

FIGURE 3: Solution effectiveness on high water content versus low water content soft contact lenses in removing Pseudomonas aeruginosa.

comparatively more effective against $P$. aeruginosa. . $^{5,14,28,29}$ Chlorhexidine found in Dymed solutions is reported as being inactive against $P$. aeruginos $a^{4}$ and their biofilms ${ }^{13}$ which may explain the large number of CFUs remaining on the lenses when treated with this solution. Resistance by microorganisms to frequently used disinfection systems, like chemical regimes, may also be a contributing factor. ${ }^{7}$ Furthermore, studies ${ }^{5,25}$ have reported poorer disinfection efficacy of solutions 
containing Dymed against $P$. aeruginosa when compared to a Polyquad solution consistent with the findings of the current study. In some instances, the Dymed solution did not achieve the required three log reduction. ${ }^{5}$ In contrast, an earlier study ${ }^{26}$ reported a Dymed solution to be more effective against P. aeruginosa than the Polyquad solution tested; however, their study was not performed on contact lenses which were identified as a limitation by the researchers. Of consideration, however, are the findings of Imayasu et al. ${ }^{30}$ who reported greater adhesion of $P$. aeruginosa to epithelial cells treated with a solution containing Polyquad compared to the one containing polyhexamethylene biguanide suggesting the possibility of a different in vivo result.

Stapleton et al. ${ }^{31}$ and Hughes et al..$^{32}$ indicated that the higher efficacy of peroxide solutions is expected in the absence of neutralisation, as was the case in this study, which therefore must be interpreted with caution as neutralisation is an essential step when using peroxide disinfection systems. This is particularly so as with the one-step peroxide system, the neutralising platinum disc is introduced during the disinfection process and therefore may not show the same efficacy as found in this study. Moreover, others ${ }^{3,21,33}$ have asserted that whilst peroxide solutions may be more effective disinfection solutions, if patients store their lenses for long periods of time then this system may not be ideal as the neutralisation process renders the solution that the lens remains in storage ineffectively as saline which may allow regrowth of microbes. The neutralised solution could not be assessed in this study as it would have meant the use of different vials for the different solutions. Earlier studies which included the neutralising step, however, reported the peroxide solution to still be more effective than other solutions against Acanthamoeba ${ }^{34}$ and Acanthamoeba castellanii (A. castellanii). ${ }^{35}$ The two-step peroxide system is, therefore, hailed to be the solution of choice for disinfection of soft contact lenses and has also been found to be more effective against Acanthamoeba than one-step peroxide systems; however, the method is less popular because of the additional step for neutralisation required..$^{3,21}$

This study was conducted on new (unworn) lenses, hence factors like lens deposits and tear components which facilitate the formation of a biofilm and thereby increase bacterial adhesion were not considered in this study. ${ }^{5}$

Efficacy may thus prove differently on used lenses as the ability of the solution to breakdown the biofilm will also be tested, particularly as the biofilm produced by P. aeruginosa has been reported to be fairly difficult to remove. ${ }^{14}$ However, Dutta et al. ${ }^{5}$ revealed, in a systematic review, that $P$. aeruginosa shows greater adhesion to unworn silicone hydrogel lenses compared to Staphylococcus microorganisms.

Whilst water content and surface charge are some of the factors reported to influence bacterial adhesion to contact lenses, ${ }^{15,22,35}$ the relative efficacy of the solutions against $P$. aeruginosa was not influenced by either water content or ionicity in the current study. Of interest though is the finding by Kierl and Christie ${ }^{18}$ that high water content ionic materials undergo changes in lens parameters when soaked overnight in $\mathrm{H}_{2} \mathrm{O}_{2}$ without neutralisation. Thus, when selecting a contact lens solution, the disinfection ability must also be weighed against toxicity of the solution as compounds from the solution can be transferred to the lens or the eye.

Disinfection efficacy also appears to be organism dependant in that the different disinfection systems have been found to have varied effectiveness on different microorganisms. ${ }^{5}$ Whilst the current study revealed the Polyquad disinfection system to be more effective against $P$. aeruginosa than the one containing Dymed, Niszl and Markus ${ }^{34}$ and Hume et al. ${ }^{36}$ found that solutions with Dymed were more effective against Acanthamoeba and Serratia marcescens (S. marcescens), respectively, when compared to Polyquad solutions. Anecdotal reports suggest that the disinfection system chosen by practitioners may be based on familiarity and convenience rather than considering effectiveness of solutions in relation to the risk profile of their patient.

Multipurpose solutions appear to be the preferred contact lens disinfection system ${ }^{7,14}$ possibly because of the ease of use, as well as cost whilst peroxide systems are often seen to be inconvenient to use and relatively expensive. Whilst multipurpose solutions may provide disinfection of a broad spectrum of microorganisms, ${ }^{5}$ microorganisms are also resistant to the antimicrobial activity of certain contact lens solutions ${ }^{13}$ and contact lens practitioners should be considering this when recommending solutions particularly to contact lens wearers who present with recurrent contact lens-related infections.

Even though the current study did not report efficacy of the solutions tested in terms of the International Organization for Standardization (ISO) 14729 standalone test requirements of a 3-log reduction criteria, 5,36 it provided comparative results in terms of the overall ability of the solutions to remove $P$. aeruginosa from a contact lens surface, and provided a relative comparison. Moreover, as there are limited studies that have included peroxide-based solutions, this study adds more information in this area. This study also showed that peroxide solutions do not just reduce the number of CFUs effectively but rather eliminate them completely. It is important that some thought is given to the appropriateness of the chosen contact lens cleaning regime. This study is of particular relevance now and in the near future with the advent of newer medical technological applications of contact lenses such as the delivery of drugs in patients with systemic conditions and smart (digitalintegrated) contact lenses being developed for interactions with an evolving digital world which will increase the popularity and usage of such contact lenses.

\section{Conclusion}

Solutions containing $\mathrm{H}_{2} \mathrm{O}_{2}$ and Polyquad appear to be most effective in removing $P$. aeruginosa from hydrogel and silicone hydrogel contact lenses, whilst those containing Dymed appear to be ineffective in removing this microorganism. 
Hydrogen peroxide solutions should therefore be considered in contact lens wearers prone to microbial keratitis. The ionicity and water content of a silicone hydrogel lens do not influence the disinfection efficacy of solutions against $P$. aeruginosa.

\section{Acknowledgements}

The authors thank Bausch and Lomb, and Cooper Vision for the sponsorship of the contact lenses.

\section{Competing interests}

The authors declare that they have no financial or personal relationships that may have inappropriately influenced them in writing this article.

\section{Authors' contributions}

R.K., S.J., J.H., T.L., S.M., S.N., K.O., M.R. and T.C. contributed to the design and implementation of the research, to the analysis of the results and to the writing of the manuscript.

\section{Funding information}

The authors received no financial support for the research, authorship and or publication of this article.

\section{Data availability}

The data that support the findings of this study are available from the corresponding author, R.H., upon reasonable request.

\section{Disclaimer}

The views expressed in the article are those of the authors.

\section{References}

1. Nichols JJ. Continuing upward trends in daily disposable prescribing and other segments maintained a healthy industry. Cont Lens Spectrum [serial online]. 2018 [cited 2020 May 14];33:20-25. Available from: https://www.clspectrum.com/ issues/2018/january-2018/contact-lenses-2017

2. Tellakula P. Care and maintenance of contact lens - An overview. Kerala J Ophthalmol. 2009;XXI:294-303.

3. Willcox M. Hydrogen peroxide versus multipurpose disinfecting solutions, what are the differences? [homepage on the Internet]. Silicone hydrogels: Editorial. 2008 [cited 2018 Aug 22]. Available from: http://www.siliconehydrogels.org/ editorials/jul_08.asp

4. McDonnell G, Russell AD. Antiseptics and disinfectants: Activity, action, and resistance. Clin Microbiol Rev. 1999;12(1):147-179. https://doi.org/10.1128/ CMR.12.1.147

5. Kal A, Toker MI, Kaya S. The comparison of antimicrobial effectiveness of contact lens solutions. Int Ophthalmol. 2017;37:1103-1114.https://doi.org/10.1007/ s10792-016-0375-4

6. Gopinathan U, Stapleton F, Sharma S, et al. Microbial contamination of hydroge contact lenses. J Appl Microbiol. 1997;82(5):653-658. https://doi. org/10.1111/j.1365-2672.1997.tb03598.x

7. Lakkis C, Fleiszig SMJ. Resistance of Pseudomonas aeruginosa isolates to hydrogel contact lens disinfection correlates with cytotoxic activity J Clin Microbiol. 2001;39(4):1477-1486. https://doi.org/10.1128/JCM.39.4.1477-1486.2001

8. Karpecki PM, Shechtman DL. Why we're stuck on Pseudomonas [homepage on the Internet]. Review of Optometry Website. November 19, 2012 [cited 2016 Feb 22]. Available from: http://www.reviewofoptometry.com/content/d/research review/c/37831

9. Amiri MA, Mohammadinia M, Tabatabaee $M$, et al. Comparative efficacies of contact lens disinfecting solutions against Pseudomonas aeruginosa. Clin Exp Optom. 2011;94(4):348-351. https://doi.org/10.1111/j.1444-0938.2010.00576.x
10. Willcox MD, Holden BA. Contact lens related corneal infections. Biosci Rep. 2001;21(4):445-461. https://doi.org/10.1023/A:1017991709846

11. Marshall KC. Interfaces in microbial ecology [homepage on the Internet]. Cambridge, MA: Harvard University Press, 1976 [cited 2020 May 13]; p. 156. Current Contents. 1992;4:156. Available from: http://garfield.library.upenn.edu/ classics1992/A1992GY20600001.pdf

12. Duran JA, Refojo MF, Gipson IK, et al. Pseudomonas attachment to new hydrogel contact lenses. Arch Ophthalmol. 1987;105(1):106-109. https://doi.org/10.1001/ archopht.1987.01060010112041

13. Szczotka-Flynn LB, Imamura Y, Chandra J, et al. Increased resistance of contact lens related bacterial biofilms to antimicrobial activity of soft contact lens care solutions. Cornea. 2009;28(8):918-926. https://doi.org/10.1097/ICO.0b013e3181a81835

14. Artini M, Cellini A, Scoarughi GL, et al. Evaluation of contact lens multipurpose solutions on bacterial biofilm development. Eye Cont Lens. 2015;41(3):177-182. https://doi.org/10.1097/ICL.0000000000000105

15. Dutta D, Cole N, Willcox M. Factors influencing bacterial adhesion to contact lenses. Mol Vis. 2012;18:14-21.

16. Stapleton F, Dart JKG, Seal DV, et al. Epidemiology of Pseudomonas aeruginosa keratitis contact lens wearers. Epidemiol Infect. 1995;144(3):395-402. https:// doi.org/10.1017/S0950268800052109

17. Bennet ES, Weissman BA. Clinical contact lens practice. Philadelphia, PA: Lippincott Williams \& Wilkins; 2005.

18. Keirl A, Christie C. Clinical optics and refraction: A guide for optometrists, contact lens opticians and dispensing opticians. Oxford: Elsevier Butterworth-Heinemann; 2007.

19. Fonn $D$, Dumbleton $K$, Jones $L$, et al. Silicone hydrogel material and surface properties [homepage on the Internet]. Cont Lens Spectrum. 2002 [cited 2018 Aug 25]. Available from: https://www.clspectrum.com/issues/2002/march-2002/ silicone-hydrogel-material-and-surface-properties

20. Seitz ME, Wiseman ME, Hilker I, et al. Influence of silicone distribution and mobility on the oxygen permeability of model silicone hydrogels. Polymer. 2017;118:150-162. https://doi.org/10.1016/j.polymer.2017.04.061

21. Hiti K, Walochnik J, Faschinger $C$, et al. One- and two-step hydrogen peroxide contact lens disinfection solutions against Acanthamoeba: How effective are they? Eye. 2005;19:1301-1305. https://doi.org/10.1038/sj.eye.6701752

22. Miller MJ, Ahearn DG. Adherence of Pseudomonas aeruginosa to hydrophilic lenses and other substrata. J Clin Microbiol. 1987;25(8):1392-1397. https://doi. org/10.1128/JCM.25.8.1392-1397.1987

23. Luensman D, Jones L. Protein deposition on contact lenses: the past, the present and the future. Cont Lens Anterior Eye. 2012;35(2):53-64. https://doi. org/10.1016/j.clae.2011.12.005

24. Dada VK, Mehta MR. Sterilization potential of contact lens solutions. Indian J Ophthalmol. 1988;36(2):92-94.

25. Codling CE, Maillard JY, Russell AD. Performance of contact lens disinfecting solutions against Pseudomonas aeruginosa in the presence of organic load. Eye Cont Lens. 2003;29(2):100-102. https://doi.org/10.1097/01.ICL.0000062347.66975.F1

26. Manuj K, Gunderson C, Troupe J, et al. Efficacy of contact lens disinfecting solutions against Staphylococcus aureus and Pseudomonas aeruginosa. Eye Cont Lens. 2006;32(4):216-218. https://doi.org/10.1097/01.icl.0000194922. 53171.39

27. Siddiqui R, Lakhundi S, Khan NA. Status of the effectiveness of contact lens solutions against keratitis-causing pathogens. Cont Lens Anterior Eye. 2015;38(1):34-38. https://doi.org/10.1016/j.clae.2014.09.001

28. Ifejika CP, McLaughlin-BorlaceL, Lucas VJ, et al. Efficacy of a contact lens cleaning device and its enhancement of the performance of contact lens care products. $\mathrm{Br}$ J Ophthalmol. 2000;84(5):539-541. https://doi.org/10.1136/bjo.84.5.539

29. Pinna A, Usai D, Zanetti $S$, et al. Susceptibility of various corneal fungal isolates and Pseudomonas aeruginosa to contact lens disinfecting solutions. J Infect Dev Ctries. 2013;7(3):261-268. https://doi.org/10.3855/jidc.2756

30. Imayasu M, Shimizu H, Shoichi BS, et al. Effects of multipurpose contact-lens care solutions on adhesion of Pseudomonas aeruginosa to corneal epithelial cells. Eye Cont Lens. 2009;35(5):98-104. https://doi.org/10.1097/ICL.0b013e3181b4d152

31. Stapleton F, Harmis N, Deshpande R, et al. Preliminary studies on the amoebicidal efficacy of contact lens disinfection systems. Aust New Zeal J Ophthalmol. 1998;26(S1):S44-S46. https://doi.org/10.1111/j.1442-9071.1998.tb01369.x

32. Hughes R, Andrew PW, Kilvington S. Enhanced killing of acanthamoeba cysts with a plant peroxidase-hydrogen peroxide-halide antimicrobial system. Appl Environ Microbiol. 2003;69(5):2563-2567. https://doi.org/10.1128/AEM.69.5.25632567.2003

33. Gray TB, Cursons RTM, Sherwan JF, et al. Acanthamoeba, bacterial, and fungal contamination of contact lens storage cases. Br J Ophthalmol. 1995;79(6): 601-605. https://doi.org/10.1136/bjo.79.6.601

34. Niszl IA, Markus MB. Anti-acanthameba activity of contact lens solutions. $\mathrm{Br}$ Ophthalmol. 1998;82(9):1033-1038. https://doi.org/10.1136/bjo.82.9.1033

35. Reverey JF, Fromme $R$, Leippe $M$, et al. In vitro adhesion of Acanthamoeba castellanii to soft contact lenses depends on water content and disinfection procedure. Cont Lens Anterior Eye. 2014;37(4):262-266. https://doi. org/10.1016/j.clae.2013.11.010

36. Hume EBH, Zhu H, Cole N, et al. Efficacy of contact lens multipurpose solutions against Serratia marcescens. Optom Vis Sci. 2007;84(4):316-332. https://doi. org/10.1097/OPX.0b013e3180465543 